\title{
Hydroxychloroquine's Probable Protective Effect in Liver injury among the COVID-19 Patients
}

Ali Reza Sima ${ }^{1, *}$

1. Digestive Disease Research Center, Digestive Disease Research Institute, Tehran University of Medical Sciences, Tehran, Iran

* Corresponding Author:

Ali Reza Sima, MD

Assistant Professor of Medicine, Digestive Diseases Research Institute, Tehran University of Medical Sciences, Shariati Hospital,

Kargar Shomali Avenue, Tehran, Iran

Tel: + 982182415104

Fax: + 982182415400

Email: simaalireza@gmail.com

Received: 25 Jul. 2020

Accepted: 06 Oct. 2020
Please cite this paper as:

Sima AR. Hydroxychloroquine's Probable Protective Effect in Liver injury among the COVID-19 Patients. Middle East J Dig Dis 2021;13:80-81. doi: 10.34172/ mejdd.2021.209.

\section{INTRODUCTION}

Coronavirus disease 2019 (COVID-19) outbreak caused by severe acute respiratory syndrome coronavirus 2 (SARS-CoV-2) has become a global emergency. The majority of the respective studies are focused on respiratory complications. However, COVID-19 might affect other organs namely the liver and kidneys. Liver damage caused by other species of the coronavirus family has been reported in the literature. ${ }^{1,2}$ Evidence of liver damage and elevated liver enzymes are also present in the studies of numerous COVID-19 patients. While clinically significant liver injury has not been reported in COVID-19 patients without underlying liver diseases, various studies have shown higher aminotransferase levels in many COVID-19 patients who had impaired baseline liver function tests (LFTs) and no known history or present symptoms of liver diseases, as well as critically ill patients..$^{3-7}$

Hypoxic hepatitis $(\mathrm{HH})$ is a common phenomenon in ICU-admitted patients. Anoxic damage of centrilobular liver tissue during $\mathrm{HH}$ causes a rapid and short-lasting influx of serum aminotransferases. Besides, preexisting cardiopulmonary failure and toxic-septic shock are the primary conditions responsible for most of the cases. Seemingly, this is one of the possible causes of elevated liver enzymes in severe ICU-admitted COVID-19 patients.

Having contradictory roles, autophagy has an irrefutable role in liver diseases. On the one hand, acting as an easily-enabled cytoprotective system, autophagy is activated by numerous causes and plays an important role in various liver diseases, but on the other hand, it can be protective during hepatic ischemia/reperfusion injury. ${ }^{8}$

Chloroquine (CQ) is a classic antimalarial drug that has been repurposed as an immunomodulator in Rheumatology. Recently, CQ is introduced and suggested as a possible beneficial drug for the management of COVID-19 patients. Chloroquine is well-known as an inhibitor of autophagy and a drug with anti-inflammatory properties. In a study published in June 2013, Fang et al demonstrated that after one hour of warm ischemia, chloroquine reduces liver 
injury within the first 6 hours after administration, but aggravates it 1 or 2 days after liver reperfusion. ${ }^{9}$ Acting like a double-edged sword, while chloroquine could protect the liver during ischemia/reperfusion injury through the inhibition of inflammatory responses in the early hours after administration, it could later aggravate liver damage via inhibition of autophagy and the resultant induction of apoptosis. ${ }^{10}$ These different effects of chloroquine on the liver injury at different times signify the value of repeated LFTs after the diagnosis and treatment initiation. Liver function tests in the recent literature could be reported from previously referred and potentially treated with hydroxychloroquine patients who have received the drug just hours before sampling (especially in the outpatient management of COVID-19). Thus, the protective effect of chloroquine on ischemic hepatitis may have masked the hepatic liver injury of coronavirus infection. To avoid misleading paraclinical data and identify the possible liver damage in COVID-19 pneumonia, serial liver function tests at close intervals are recommended.

Emphasizing on chloroquine's inhibitory effects on HMGB1-mediated inflammatory responses and its facilitatory effects on pro-apoptotic pathways, another study has shown chloroquine's possible clinical utility for acute liver injury. As liver injury carries on, chloroquine could regulate the resultant apoptotic and inflammatory responses. ${ }^{11}$

We suspect that the probable protective effects of prophylactic hydroxychloroquine for the liver might be helpful to reduce the incidence of potential liver failure in COVID-19 patients with hepatic comorbidities.

\section{ETHICAL APPROVAL}

There is nothing to be declared.

\section{CONFLICT OF INTEREST}

The authors declare no conflict of interest related to this work.

\section{REFERENCES}

1. Bangash MN, Patel J, Parekh D. COVID-19 and the liver: little cause for concern. Lancet Gastroenterol Hepatol 2020;5:529-30. doi: 10.1016/S2468-1253(20)30084-4.

2. Yip TC, Lui GC, Wong VW, Chow VC, Ho TH, Li TC, et al. Liver injury is independently associated with adverse clinical outcomes in patients with COVID-19.
Gut 2020;gutjnl-2020-321726. doi: 10.1136/ gutjnl-2020-321726. Online ahead of print.

3. Wijarnpreecha K, Ungprasert P, Panjawatanan P, Harnois DM, Zaver HB, Ahmed A, et al. COVID-19 and liver injury: a meta-analysis. Eur J Gastroenterol Hepatol 2020 Jul 3. doi: 10.1097/MEG.0000000000001817. Online ahead of print.

4. Kumar MP, Mishra S, Jha DK, Shukla J, Choudhury A, Mohindra R, et al. Coronavirus disease (COVID-19) and the liver: a comprehensive systematic review and metaanalysis. Hepatol Int 2020;14:711-22. doi: 10.1007/ s12072-020-10071-9.

5. Kulkarni AV, Kumar P, Tevethia HV, Premkumar M, Arab JP, Candia R, et al. Systematic review with meta-analysis: liver manifestations and outcomes in COVID-19. Aliment Pharmacol Ther 2020;52:584-99. doi: 10.1111/ apt.15916.

6. Jiang S, Wang R, Li L, Hong D, Ru R, Rao Y, et al. Liver Injury in Critically Ill and Non-critically Ill COVID-19 Patients: A Multicenter, Retrospective, Observational Study Front Med (Lausanne) 2020;7:347. doi: 10.3389/ fmed.2020.00347. eCollection 2020.

7. Fu T, Deng M, Wang X, Yang F. Predictor of poor prognosis of COVID-19 patients----liver injury. Expert Rev Gastroenterol Hepatol 2020;14:873-76. doi:10.1080/174 74124.2020.1793670.

8. Klionsky DJ, Emr SD. Autophagy as a regulated pathway of cellular degradation. Science 2000;290:1717-21. doi: 10.1126/science.290.5497.1717.

9. Fang H, Liu A, Dahmen U, Dirsch O. Dual role of chloroquine in liver ischemia reperfusion injury: reduction of liver damage in early phase, but aggravation in late phase. Cell Death Dis 2013;4:e694. doi: 10.1038/cddis.2013.225.

10. Jiang M, Liu K, Luo J, Dong Z. Autophagy is a renoprotective mechanism during in vitro hypoxia and in vivo ischemia-reperfusion injury. Am J Pathol 2010;176:118192. doi: 10.2353/ajpath.2010.090594.

11. Dai C, Xiao X, Li D, Tun S, Wang Y, Velkov T, et al. Chloroquine ameliorates carbon tetrachloride-induced acute liver injury in mice via the concomitant inhibition of inflammation and induction of apoptosis. Cell Death Dis 2018;9:1164. doi: 10.1038/s41419-018-1136-2. 